\title{
ANN Optimized by ICSA Used in Fault Diagnostics
}

\author{
Zhenguo Chen \\ Department of Computer Science and Technology \\ North China Institute of Science and Technology \\ East Yanjiao, Beijing 101601, China \\ e-mail: zhenguo_chen@126.com
}

\author{
Xiaoju Wang \\ Department of Computer Science and Technology \\ North China Institute of Science and Technology \\ East Yanjiao, Beijing 101601, China \\ e-mail: wangrace@ncist.edu.cn
}

\author{
Liqin Tian \\ Department of Computer Science and Technology \\ North China Institute of Science and Technology \\ East Yanjiao, Beijing 101601, China \\ e-mail: tianliqin@ncist.edu.cn
}

\begin{abstract}
Fault diagnostics is to distinguish the current state of the equipment is in normal or abnormal. It can be seen as a problem of multi-class classification. To improve the performance of classification, this paper presents a novel method for fault diagnosis. In this method, we synthetically applied immune clonal selection algorithm and artificial neural network technology to the fault diagnosis of steamturbine generator. The method consists of two stages. Firstly, the parameters of artificial neural network were optimized by immune clonal selection algorithm. Then an artificial neural network classifier with parameter optimization is constructed and used to identify the fault of steam-turbine generator. The experimental result using the a steam turbine fault diagnosis dataset shows that the fault diagnostics based on artificial neural network optimized by immune clonal selection algorithm can give higher recognition accuracy than other traditional methods.
\end{abstract}

Keywords-component; Fault diagnostics; Artificial neural network; Immune clonal selection; True detection rate; parameter optimization

\section{INTRODUCTION}

Fault detection and diagnosis in manufacturing processes is of great practical significance. Fault detection is recognizing that a problem has occurred, even if you don't yet know the root cause. Faults may be detected by a variety of quantitative or qualitative means. This includes many of the multivariable, model-based approaches discussed later. It also includes simple, traditional techniques for single variables, such as alarms based on high, low, or deviation limits for process variables or rates of change.

Recently, the general problem of fault diagnosis has been getting more attention. A lot of researches have been applied in the field of fault diagnosis. Reza has designed a hierarchical neural network based on fuzzy clustering for fault diagnosis of the Tennessee-Eastman process[2]. Jack and Nandi used support vector machines for detection and characterisation of rolling element bearing faults[3].
Kankar et al. Investigated the use of machine learning method for fault diagnosis of ball bearing[4].Perk and Shao et al. gives an adaptive fault-tolerant control framework [5]. Sujatha and Pappa introduced the GA and SVM into non linear controllers in batch reactor[6].Xu et al. present an ensemble method for process fault diagnosis[7]. Muhammet et al. propose artificial neural network (ANN) based fault estimation algorithm was verified with experimental tests and promising results [8].In addition, there are some other combination method is proposed [9-11] and have been used effectively to detect the machine faults in the past few years.

In neural network applications, we will inevitably face a problem that the network structure and other parameters lack the available theory guarantee. We must have a great effect on the practical capability and efficiency of neural network model.

According to above problems, we must choose an appropriate optimum algorithm to optimize the parameters of ANN. Immunity Clonal Selection Algorithm [1] (ICSA) is a new intelligent algorithm which integrates global search with local search, and can effectively overcome the prematurity and slow convergence speed of traditional evolution algorithm. So Immune Clonal Selection Algorithm is a proper method of parameter optimization. All of these mentioned above, Fault diagnostics based on artificial neural networks with parameter optimization by immune clonal selection is proposed in the paper.

The remainder of the paper is organized as follows: In Section 2, we give a brief introduction to Immunity Clonal Selection Algorithm [1] used in this paper. In section 3, describe the model establishment. In Section 4, describes simulation and analysis of results. Finally, we conclude the paper in Section 5.

\section{BRIEF INTRODUCTION TO IMMUNITY CLONAL SELECTION ALGORITHM}

It was Burnet who originally proposed the famous clonal selection principle in 1958. The principle describes the basic features of an immune response to an antigenic 
stimulus. It establishes the idea that only those cells that recognize the antigens proliferate, thus being selected. When exposed to antigens, immune cells that may recognize and eliminate the antigens can be selected in the body and mount an effective response against them during the course of the clonal selection.

The main steps are as following:

Step1: $\mathrm{k}=0$, initialize the antibody population, set the algorithm's parameters and calculate the affinities of the initializing population;

Step2: According to the affinities and the given integer relating to the scale of the clone, carry out the clonal operation, clonal mutation operation and clonal selection operation; obtain the new antibody population;

Step3: Calculate the affinities of the current population;

Step4: $\mathrm{k}=\mathrm{k}+1$ if a stop condition is satisfied, stop the algorithm, else go to step2

\section{MODEL ESTABLISHMENT}

\section{A. Determination of the encoding scheme}

The first step, when Immunity Clonal Selection Algorithm [1] (ICSA) is applied, is the determination of an encoding scheme, namely to denote each possible point in the problem's search space as a characteristic string of defined length. This is in order to be sure that immunity clonal selection algorithm (ICSA) will not only optimize network configuration but, in the meantime, Immunity Clonal Selection Algorithm (ICSA) training will proceed on weight values. In this paper, weight values between each layer of the multi-layer feed-forward neural network are simultaneously coded as one chromosome. Fig .1 show our encoding scheme.

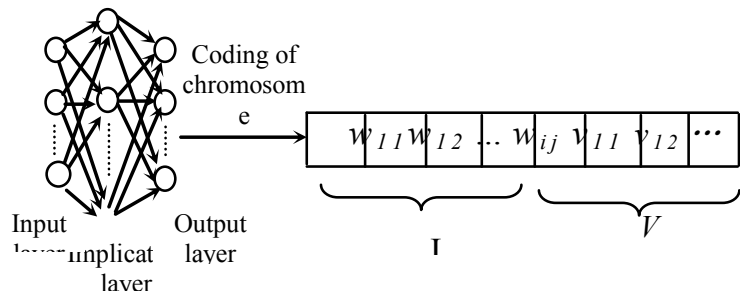

Figure 1. Neuron network structure and coded chromosome

\section{B. Definition of flexibility function}

The second key step is the definition of the flexibility function to evaluate the problem-solving ability of the neural network, which is denoted by a certain specific chromosome string. In this paper, objective function is generated from the cost factor mean-square error (MSE) of the neural network output, and then converted into a function via reciprocal transformation. Its computational formula is as follows:

$$
M S E=\frac{1}{m p} \sum_{P=1}^{p} \sum_{j=1}^{m}\left(d_{p j}-y_{p j}\right)^{2}
$$

Where $\mathrm{m}=$ sum of output nodes; $p=$ sum of trained samples; $d_{p j}=$ expected output of network; $y_{p j}=$ actual output of network.
So we designed the affinity function as:

$$
f(M S E)=(S+1) /(M S E+1)
$$

In which the selection of $S$ is quite crucial, as it determines the coerciveness of the selection procedure. $S$ is determined from experiments.

\section{Three of Clonal Selection elemental operators}

\section{1) Clonal operation}

$T_{c}^{C}(\stackrel{\vee}{A}(k))=\left[T_{c}^{C}\left(A_{1}(k)\right) \quad T_{c}^{C}\left(A_{2}(k)\right) \quad \mathrm{L} \quad T_{c}^{C}\left(A_{n}(k)\right)\right]^{T}$

Where: $T_{c}^{C}\left(A_{i}(k)\right)=I_{i} \times A_{i}(k) i=1,2, \ldots, \mathrm{n}$

$$
q_{i}=\operatorname{Int}\left(N_{c} * \frac{f\left(A_{i}(k)\right)}{\sum_{j=1}^{n} f\left(A_{j}(k)\right)}\right)
$$

Where $N_{c}>\mathrm{n}$ is a given integer relating to the clonal scale. We set $N_{c}=20$ herein. $\operatorname{Int}(x)$ represents the minimum integer which is bigger than $x$. Formula(1) shows that clonal scale adjusts adaptively according to the affinity between antibody and antigen. After clonal operation, the antibody population changes into formula (4):

$$
\stackrel{A^{\prime}}{ }(k)=\left\{\stackrel{v}{A}(k), \stackrel{\mathrm{v}}{A_{1}^{\prime}}(k), \stackrel{\mathrm{v}}{A_{2}^{\prime}}(k), \mathrm{L}, \stackrel{\mathrm{v}}{A_{n}^{\prime}}(k)\right\}
$$

\section{2) Clonal mutation operation}

Unlike the general mutation operator in GA, in order to save the information of the original population, the clonal mutation is unused to $A \in A^{\prime}$.

We carry out mutation operation on the current population which we get from the clonal operation according to the given probability of $p_{m}^{i}$,

$$
\bar{A}^{\prime}(k)=T_{g}^{C}(\bar{A}(k)) \text {. }
$$

In this paper we set the probability of mutation as 0.5 .

$$
p_{s}= \begin{cases}1 & f\left(\mathrm{~A}_{i}(k)\right)<f(B) \\ \exp \left(-\frac{f\left(A_{i}(k)\right)-f(B)}{\alpha}\right) & f\left(A_{i}(k)\right) \geq f(B) \text { and } \mathrm{A}(\mathrm{k}) \text { is the } \\ & \text { best antibody in the population } \\ & f\left(A_{i}(k)\right) \geq f(B) \text { and } \mathrm{A}(\mathrm{k}) \text { is not } \\ & \text { the best antibody in the population }\end{cases}
$$

\section{3) Clonal selection operation}

$\forall \mathrm{i}=1,2, \ldots$, , if existing the antibody after mutation: $\mathrm{B}=\left\{A_{i j}^{\prime}(k) \mid \max f\left(A_{i j}^{\prime}\right), j=1,2, \ldots, \mathrm{qi}-1\right\}$, then the probability that B replaces the $A_{i}(k) \in \bar{A}(k)$ is as formula (6). 


\section{Fault diagnosis model based on ICSA+ANN}

In this section, we provide a definition of the fault diagnosis model based on artificial neural networks with parameter optimization by immune clonal selection algorithm. As shown in Fig .2.

In this model, we choose the Steam-turbine generator data as the experimental data set and the data set is divided into two parts: training data set and test data set.

Then, through the continuous experiments in data set, use the ICS algorithm for optimization of ANN algorithm, has obtained the optimized parameter values.

Thirdly, we construct a parameter optimization neural network classifier is used in classifying effect on classifier, and the method is verified by the test data, to make the appropriate fine-tuning according to the verification results

Finally, fault diagnosis using the constructed classifier, and actual results.

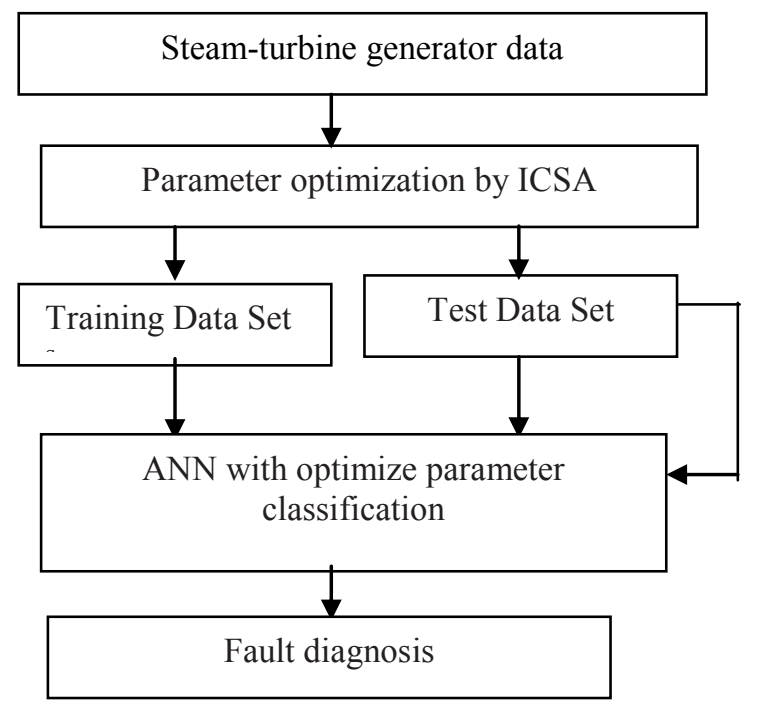

Figure 2. Model of fault diagnosis procedure

\section{SimUlations AND RESUlts ANALYSiS}

\section{A. Data set used in the experiments}

To demonstrate the effectiveness of the proposed ICSA-ANN in fault diagnosis, the experiments are conducted on a steam turbine fault diagnosis dataset. Rotor unbalance is a familiar fault phenomenon of large machines. To discover the fault as early and accurate as possible, the possibility of occurrence of fault should be evaluated based on the symptom of vibrancy signal. The original data are shown in Table I, in which S1 S11 are the symptoms and $\mathrm{D}$ is the decision.

\section{B. Experiment results and analysis}

To be clearer, we use two performance measures in our experiments. The true detection rate (TDR) measures the percentage of correctly classified in the test set. The false detection rate (FDR) measures the percentage of classified in the test set that are detected false.

We have performed simulation using BPNN, a discrete event simulator. $\mathrm{BP}+$ Moentum and the proposed defending model TBDM-GR in this paper are respectively implemented in simulation. Each method was tested on the same suite of data sets, and the comparison of the following three algorithms' results are showed in Table II, each result is the statistical mean value generated by 100 times calculations separately.

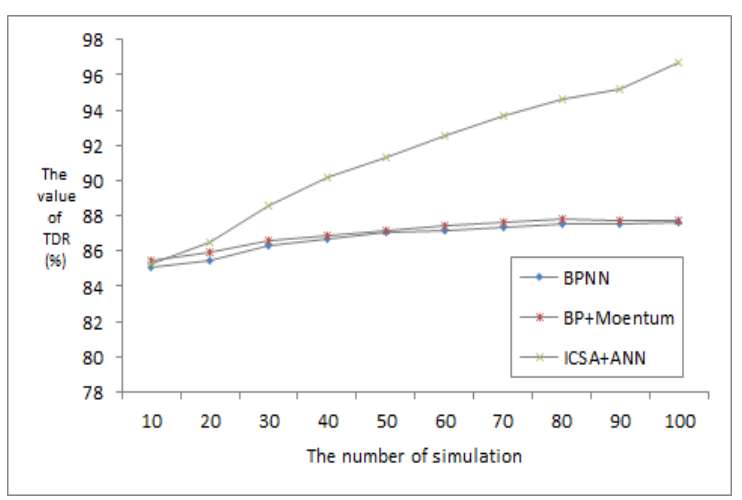

Figure 3. the statistical mean value of TDR

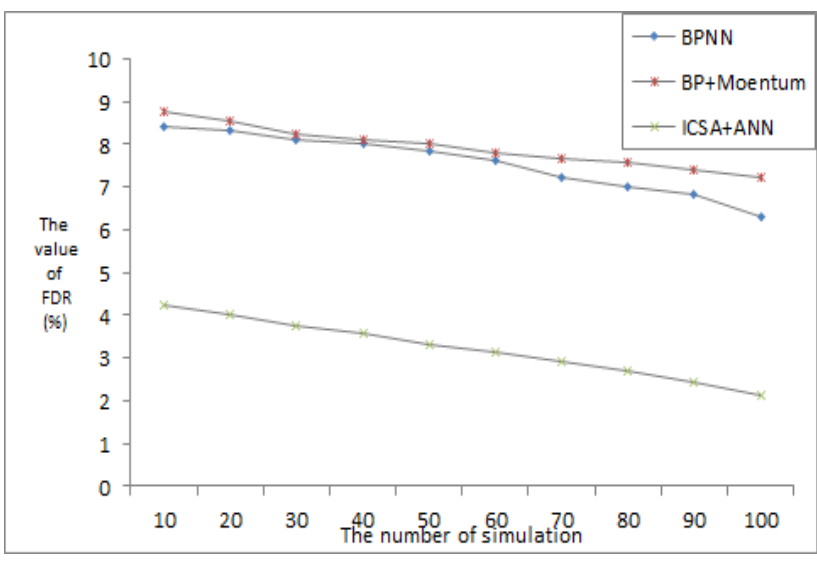

Figure 4. the statistical mean value of FDR

TABLE I. ClassiC INPUT AND OUTPUT SAMPLES

\begin{tabular}{ccccccccccccc}
\hline No. & S1 & S2 & S3 & S4 & S5 & S6 & S7 & S8 & S9 & S10 & S11 & D \\
\hline 1 & 0.8 & 0 & 0.1 & 0.1 & 1 & 0 & 1 & 0.1 & 0.9 & 1 & 0 & 1 \\
2 & 0.8 & 0 & 0.1 & 0.1 & 0.8 & 0 & 0.8 & 0.1 & 0.8 & 0.9 & 0 & 1 \\
3 & 0.5 & 0 & 0.1 & 0.1 & 0.8 & 0 & 1 & 0.1 & 0.7 & 0.9 & 0 & 1 \\
4 & 0.8 & 0 & 0.2 & 0.2 & 1 & 0 & 1 & 0.1 & 0.9 & 1 & 0 & 1 \\
5 & 0.5 & 0 & 0.1 & 0.1 & 0.8 & 0 & 0.9 & 0.1 & 0.7 & 0.9 & 0 & 0 \\
6 & 0.5 & 0.9 & 0 & 0.8 & 0 & 0.8 & 0.1 & 0.9 & 0.5 & 0.1 & 0.8 & 0 \\
7 & 0.5 & 0.9 & 0 & 0.8 & 0 & 0.8 & 0.1 & 0.5 & 0.5 & 0.2 & 0.9 & 0 \\
8 & 0.6 & 0.7 & 0 & 0.9 & 0 & 0.5 & 0.1 & 0.8 & 0.4 & 0.1 & 0.7 & 0 \\
9 & 0.4 & 0.7 & 0 & 0.7 & 0 & 0.5 & 0.1 & 0.7 & 0.3 & 0.1 & 0.6 & 1 \\
10 & 0.3 & 0.9 & 0 & 0.9 & 0 & 1 & 0 & 0.8 & 0.1 & 0 & 0.9 & 0 \\
11 & 0.2 & 0.7 & 0 & 0.8 & 0 & 1 & 0 & 0.8 & 0 & 0 & 0.8 & 0 \\
12 & 0.2 & 0.6 & 0 & 0.6 & 0 & 0.9 & 0 & 0.7 & 0 & 0 & 0.6 & 0 \\
13 & 0.4 & 0.4 & 0.3 & 0.6 & 0 & 0 & 0.1 & 0.1 & 0.2 & 0 & 0.1 & 0 \\
14 & 0.4 & 0.5 & 0.3 & 0.7 & 0.05 & 0 & 0 & 0.1 & 0.1 & 0 & 0.1 & 0 \\
15 & 0.4 & 0.6 & 0.4 & 0.9 & 0 & 0.8 & 0 & 0.3 & 0.1 & 0 & 0.9 & 0 \\
16 & 0.3 & 0.8 & 0.3 & 1 & 0 & 1 & 0 & 0.1 & 0 & 0 & 1 & 0 \\
17 & 0.3 & 0.4 & 0.3 & 1 & 0 & 1 & 0 & 0.1 & 0 & 0 & 1 & 0 \\
18 & 0.6 & 0.3 & 0.9 & 0.3 & 0.3 & 0 & 0 & 0 & 0 & 0 & 0.6 & 0 \\
19 & 0.7 & 0.3 & 0.9 & 0.3 & 0.2 & 0 & 0 & 0 & 0 & 0 & 0.8 & 0 \\
20 & 0.7 & 0.6 & 0.9 & 0.6 & 0 & 0 & 0.2 & 0.5 & 0.3 & 0 & 0.9 & 1 \\
21 & 0.7 & 0.6 & 0.9 & 0.7 & 0 & 0 & 0.3 & 0.6 & 0.4 & 0 & 0.8 & 1 \\
\hline
\end{tabular}


Fig .3 shows the statistical mean value of TDR in the three kinds of algorithm.

Fig .4 shows the statistical mean value of FDR in the three kinds of algorithm.

TABLE II. PERFormance OF THREE METHOdS (\%)

\begin{tabular}{|c|c|c|}
\hline \multirow{2}{*}{ Algorithms } & \multicolumn{2}{|c|}{ Test Datasets } \\
\cline { 2 - 3 } & TDR.(\%) & FDR (\%) \\
\hline BPNN & 87.60 & 6.31 \\
\hline BP+Moentum & 87.73 & 7.25 \\
\hline ICSA+ANN & 96.73 & 2.13 \\
\hline
\end{tabular}

As shown in Table II, our algorithm in the true detection rate (TDR) than BPNN and $\mathrm{BP}+$ Moentum algorithms are improved by 9.13 and 9 percentage points. The false detection rates (FDR) were reduced by 4.18 and 5.12 percentage points. Therefore, our model is effective, can obtain higher recognition accuracy and have lower FDR.

\section{CONCLUSION AND FUTURE WORK}

This paper has investigated the use of artificial neural networks with parameter optimization by immune clonal selection algorithm in the fault diagnostics and tested their performance on a set of steam-turbine generator data. As the result of these experiments, the fault diagnostics based on artificial neural networks with parameter optimization by immune clonal selection algorithm achieves the higher recognition accuracy than other method.

In addition, the model presented in this paper need further research direction, is the universal verification algorithm, because this only for steam-turbine generator data were validated, and to other types of fault diagnostics, also need to obtain the parameters of fault diagnostics.

\section{ACKNOWLEDGMENT}

The work was supported by the Fundamental Research Funds for the Central Universities (3142013098, 3142013070, 3142013074, 3142014125) and Natural Science Foundation of Hebei Province of China (F2014508028).

\section{REFERENCES}

[1] H.F. Du, L.C. Jiao. Clonal operator and antibody clone algorithms. Proceedings of 2002 International Conference on Machine Learning and Cybernetics. 2002: 506-510.

[2] Reza Eslamloueyan, Designing a hierarchical neural network based on fuzzy clustering for fault diagnosis of the Tennessee-Eastman process, Applied Soft Computing, 2011,11(1): 1407-1415.

[3] L.B. Jack, A.K. Nandi, Support vector machines for detection and characterisation of rolling element bearing faults, Journal of Mechanical Engineering Science 9 (2001) 1065-1074.

[4] P. K. Kankar, S. C. Sharma, and S. P. Harsha, Fault diagnosis of ball bearings using machine learning methods, Expert Systems with Applications, 2011,38(3): 1876-1886.

[5] S. Perk, Q.M. Shao, F. Teymour, A. Cinar, An adaptive faulttolerant control framework with agent-based systems, International Journal of Robust and Nonlinear Control, 2012, 22: 43-67.

[6] S. Sujatha and N. Pappa, "Realization of non linear controllers in batch reactor using GA and SVM," International Journal of Chemical Reactor Engineering, 2011,9(1).

[7] Y. Xu, Y.J. Chen, and Q.X. Zhu, An Extension Sample Classification-Based Extreme Learning Machine Ensemble Method for Process Fault Diagnosis. Chem. Eng. Technol, 2014(37): 911918.

[8] U. Muhammet,O. Mustafa,D. Mustafa and K. Haluk. Fault diagnosis of rolling bearings using a genetic algorithm optimized neural network, Measurement, 2014(5):187-196.

[9] H. Talhaoui, A. Menacer, R. Kechida. Rotor resistance estimation using EKF for the rotor fault diagnosis in sliding mode control induction motor, Systems and Control (ICSC), 2013 3rd International Conference on, pp: 43 - 49

[10] L. Zhang, L.B. Jack, A.K. Nandi, Fault detection using genetic programming, Mechanical Systems and Signal Processing 19 (2005):271-289.

[11] Y.S. Wang, Q.H. Ma, Q. Zhu, X.T. Liu, L.H. Zhao, An intelligent approach for engine fault diagnosis based on Hilbert-Huang transform and support vector machine, Applied Acoustics, 2014(75): 1-9. 